\title{
Aortic rupture as a result of low velocity crush
}

\author{
C Reid, S A Livesey, C V Egleston
}

\begin{abstract}
A case of aortic disruption in a 35 year old lorry driver is described. This occurred as a result of a low velocity crushing force. Clinicians should be aware that this mechanism of injury may result in aortic disruption as well as the more commonly mentioned severe deceleration force. (F Accid Emerg Med 1999;16:299-300)
\end{abstract}

Keywords: aortic disruption; crush injury

Traumatic disruption of the thoracic aorta is a potentially lethal injury. It is usual to suspect the likelihood of this injury on the basis of history, most commonly falls from a significant height or motor vehicle collisions. ${ }^{1}$ These mechanisms usually involve shearing forces brought about by rapid deceleration. We present a case of aortic disruption brought about not by rapid deceleration but by a severe

Emergency

Department,

Southampton General

Hospital, Tremona

Road, Southampton

SO16 6YD

C Reid

C V Egleston

Wessex Cardiothoracic Centre, Southampton General Hospital

S A Livesey

Correspondence to:

Dr Egleston, Consultant in Accident and Emergency

Medicine.

Accepted 31 October 1998

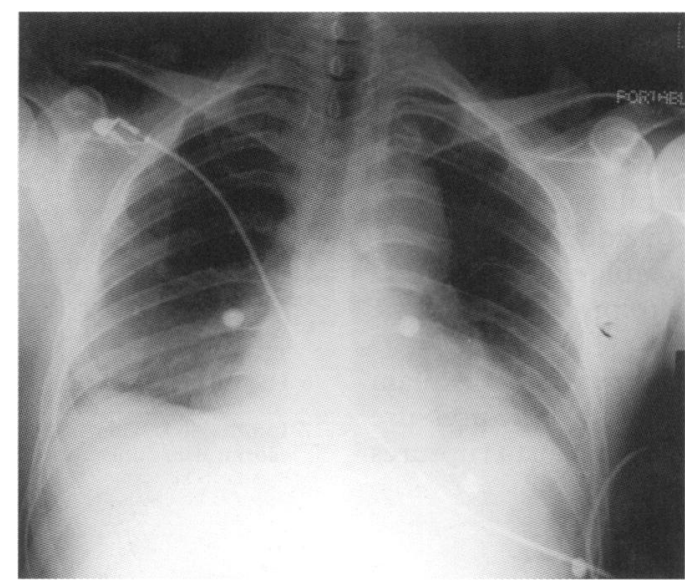

Figure 1 Semierect chest radiograph showing widened mediastinum and increased opacity of bilateral lower zones indicating bilateral haemothoraces.

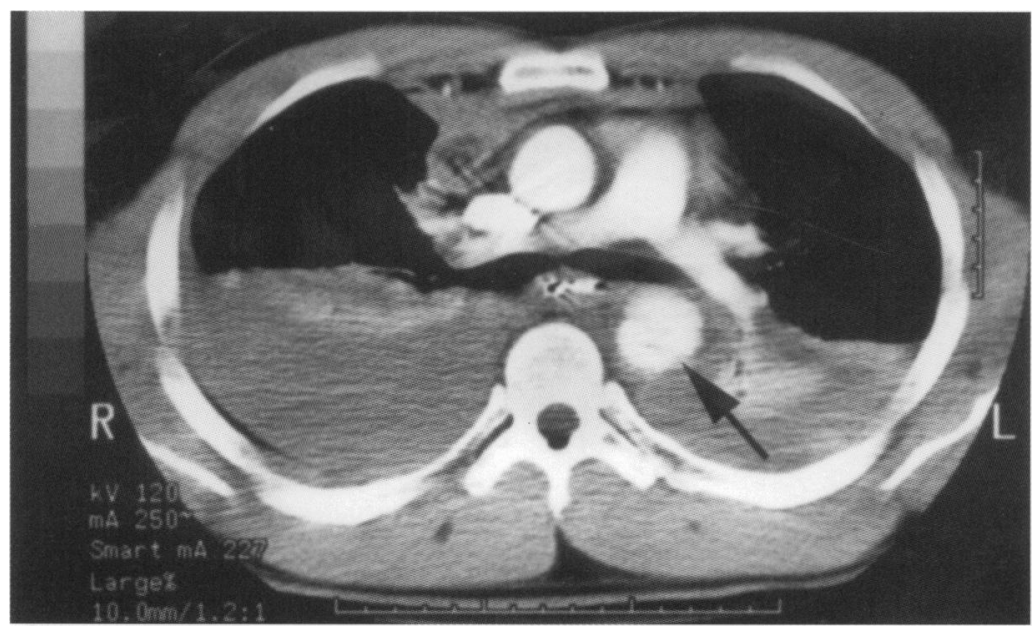

Figure 2 Computed tomography showing bilateral haemothoraces and aortic intimal tear (arrow). crushing mechanism. We also review the literature dealing with possible mechanisms of aortic disruption.

\section{Case report}

A 35 year old man was unloading his 20 tonne truck when it rolled backwards pinning him to a wall. The open tailgate crushed the area of the man's lower chest and upper abdomen. On arrival in the emergency department he was alert and orientated, had a clear airway, and complained of pain in his lower chest and upper abdomen. Examination revealed bruising of this area. He had a respiratory rate of 28 beats/min and no abnormality was detected on chest auscultation. He was pale, sweaty, had a heart rate of 140 beats/min and blood pressure of $120 / 65 \mathrm{~mm} \mathrm{Hg}$. Pulse oximetry showed an oxygen saturation of $96 \%$ on $85 \%$ inspired oxygen. The patient was unwilling to lie flat due to severe pain in his back and abdomen. A semierect chest radiograph (fig 1) was performed as an adjunct to the primary survey. On the basis of this radiograph and the abdominal findings it was thought possible that the patient had severe abdominal visceral injury and aortic disruption.

Emergent spiral computed tomography of the chest and abdomen was performed; this demonstrated bilateral haemothoraces, an anterior mediastinal haematoma, and abnormality of the aortic isthmus with at least one intimal flap and a small adjacent haematoma (fig 2). A liver laceration was also demonstrated but there was no obvious free fluid in the abdomen. Aortography was performed (fig 3) demonstrating transection of the aorta distal to the left subclavian artery. The patient had prompt surgical repair of this transection and was cared for on intensive care. Recovery was

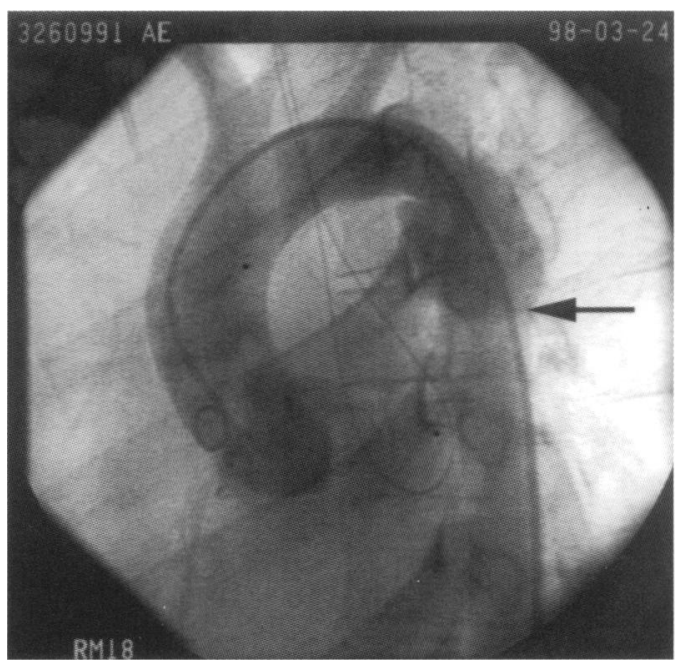

Figure 3 Arch aortogram showing transection of the aorta just distal to the left subclavian artery (arrow). 
complicated by development of a distended abdomen and pyrexia the day after aortic surgery. Laparotomy revealed blood in the peritoneal cavity, a retroperitoneal haematoma, and a contused pancreas. There was no active bleeding from the hepatic laceration. Peritoneal lavage was performed and a pancreatic drain was sited. After this the patient made a good recovery and was discharged from hospital 17 days after admission.

\section{Discussion}

Traumatic aortic disruption is a potentially devastating injury. Prompt diagnosis is required in all and prompt repair in most cases. The keys to making a rapid diagnosis are suspecting the condition from the mechanism of injury and the presence of typical findings on chest radiography, most commonly a widened mediastinum. These findings make rapid referral to a cardiothoracic surgeon and arch aortography a necessity.

There are three theories that have been put forward to explain the mechanism of blunt aortic disruption. The first theory is that at the moment of impact the heart and aorta remain stationary while the thoracic aorta moves forward ${ }^{2}$; this is thought to be improbable as a mechanism, however, on account of the descending aorta being fixed by the intercostal vessels and the ductus arteriosus. ${ }^{3}$

The second theory is that the descending aorta remains fixed to the thoracic wall while the heart and the arch of the aorta swing forward resulting in a shearing force at the level of the ductus arteriosus. This was found to be the most common site of injury in most series describing this injury. ${ }^{4-6}$ In addition to deceleration other workers postulated that direct impact was also necessary to produce an aortic tear. $^{2}$

The third theory put forward to explain blunt aortic rupture is that hydrostatic pressure rises markedly at impact and the aorta ruptures at its weakest points. Coermann et al and Lundevall produced work in support of this theory. ${ }^{47}$ Lundevall described a "water hammer" effect in which only $578 \mathrm{~g}$ to $660 \mathrm{~g}$ of force is necessary to cause aortic disruption. ${ }^{7}$ It is estimated that these forces can be generated in a vehicle crashing at $64 \mathrm{kph}(40 \mathrm{mph})$ where deceleration occurs in milliseconds. In cadaver experiments exploring the mechanism of blunt aortic rupture Coermann et al found that intraluminal pressures ranged from $300 \mathrm{~g}$ to greater than $1000 \mathrm{~g}$.

The most plausible mechanism of aortic rupture in most cases is probably a combination of the second and third theories. However in the case reported there was considerable crushing force (as evidenced by the presence of a contused pancreas and retroperitoneal haematoma on laparotomy) but no severe deceleration. It is likely that the aortic rupture in this case was caused by a violent increase in intraluminal pressure.

The best established trauma text in this country is the Advanced Trauma Life Support manual, which states that "Traumatic aortic rupture is a common cause of sudden death after an automobile collision or fall from a great height". This statement in view of this case report and other work mentioned above might well benefit from amendment to include severe crush as a possible mechanism of injury.

1 American College of Surgeons Committee on Trauma. Advanced trauma life support for doctors. 6th Ed. Chicago: American College of Surgeons, 1997.

2 Keen G. Closed injuries of the thoracic aorta. Ann $R$ Coll Surg Engl 1972;51:137-56.

3 Bowen DAL, Teare RD. Delayed traumatic rupture of aorta. Thorax 1962;17:150-3.

4 Coermann R, Dotzauer G, Lange W, et al. The effects of the design of the steering assembly and the instrument panel on injuries (especially aortic rupture) sustained by car drivers in head-on collision. $₹$ Trauma 1972;12:715-24.

5 Williams JS, Graff JA, Uku JU, et al. Aortic injury in vehicular trauma. Ann Thorac Surg 1994;57:726-30.

6 Hilgenberg AD, Logan DL, Akins CW, et al. Blunt injuries of the thoracic aorta. Ann Thorac Surg 1992;53:233-8.

7 Lundevall J. The mechanism of traumatic rupture of the aorta. Acta Pathol Microbiol Scand 1964;62:34-46. 